\title{
Nanoscale Characterization of Escherichia coli Biofilm Formed under Laminar Flow Using Atomic Force Microscopy (AFM) and Scanning Electron Microscopy (SEM)
}

\author{
Jeesun Lim, Kang-Mu Lee, So Hyun Kim, Seong-Won Nam, Yoo Jin Ol, ${ }^{\dagger}$ Hyun Sun Yun, ${ }^{\ddagger}$ \\ William Jo, ${ }^{\ddagger}$ Sejong Oh, ${ }^{\S}$ Sae-Hun Kim, ${ }^{\ddagger}$ and Sungsu Park ${ }^{*}$ \\ Department of Chemisty and Nano Sciences (BK21), Ewha Womans University; Seoul 120-750, Korea \\ "E-mail: nomopark(a) wha.ac.kr \\ ${ }^{\circ}$ Department of Physics, Euha Womans University; Seoul 120-750, Korea \\ Division of Food Science and Technologv, Korea University, Seoul 136-701, Korea \\ \$Department of Animal Science, Chomam National University, Gwangiu 500-757, Korea \\ Received July:25, 2008
}

\begin{abstract}
Biofilm contains heterogeneous three-dimensional structures composed of extracellular polymeric substance (EPS), which are greatly influenced by flow conditions. Here, we report a microfluidic platform highly suitable for nanoscale investigation of biofilms formed under laminar flows. This is possible because biofilns formed on glass beads having an average diameter of about $200 \mathrm{~m}$ in the microfluidic device can be easily taken out and located for imaging under high resolution microscopes, such as atomic force microscope (AFM) and scarning electron microscope (SEM). Escherichia coli formed biofilms in the device at various flow conditions $\left(0-50 \mu \mathrm{L} \mathrm{min}{ }^{-1}\right)$ for several days. SEM showed nanopores (14-100 $\left.\mathrm{mm}\right)$ in biofilm at lower flow rates $(0.5$ and $5 \mu \mathrm{L} \mathrm{min}^{-1}$ ) orly at within 3 days, while such small pores were not observed at higher flow rates $(50 \mu \mathrm{L}$ $\min ^{-1}$ ) during the entire culture period ( $0-5$ days). AFM results showed that the surface coverage and roughness of biofilm increased as the flow rate increased. These results suggest that like turbulent flow regime flow rates under laminar flow regime greatly influence on the morphology of biofilms in both micro- and nanoscales.
\end{abstract}

Key Words : AFM, Biofilm, Escherichia coli O157:H7, Nanostructure, SEM

\section{Introduction}

Biofilm has three-dimensional (3D) structure of bacterial cells enclosed in a self-produced extracellular polymeric substance (EPS) and adherent to an inert or living surface. ${ }^{1}$ Biofilm develops naturally and exists predominantly in most natural environments. ${ }^{2}$ Biofilm causes a wide variety of microbial infections in the body, by estimate $65 \%$ of all infections. ${ }^{3}$ Because bacteria embedded within biofilm can be 10-1,000 times more resistance to detergent, antibiotics and host defense mechanisms than their planktonic counterparts. ${ }^{+}$

Biofilms formed under fluidic conditions have different morphological characteristics such as structure, density and behavior from those formed in static conditions" Furthermore, their response to the action of antimicrobial agents depends on the velocity filed of the fluid in contact with the microbial layer. ${ }^{6}$ Consequently, many researchers developed diverse methodologies for biofilm monitoring and studied about the effect of hydrodynamic conditions on biofilm to understand and regulate the mechanical properties of biofilm. The capillary tube system is widely used for morphological investigation of biofilm under controlled fluidic condition with confocal laser scanning microscope (CLSM). ${ }^{7}$ However, the difficulty of high-resolution investigation limits its use for study of nanoscale structures. ${ }^{8,4}$ It is not suited to introduce sample into microscopes that directly observe surface with high-resolution such as scanning electron microscope (SEM) and atomic force microscope (AFM), because biofilm is fixed inside tubes. ${ }^{10,11}$

Herein, we introduced microfluidic platform that made possible the micro- and nanoscales investigation of biofilm formed under laminar flow regimes. Microfluidic device is ideally suitable for biofilm research because it allows the precise control of physiological flow velocities, low fluid-tocell volume ratios and use of small amount of resources. ${ }^{12.13}$ The device described here had large surface and gradually increased area to facilitate microbial adhesion and prevent to backward flows of bacteria. First of all, the advantage of this device was the use of glass beads with large diameter of 150 $212 \mu \mathrm{m}$. Glass beads offered large surface area-to-volume ratio for higher binding chance..$^{14}$ In addition, straightforward procedure to acquire glass beads from microchannel was possible to observe biofilms under diverse microscopes such as SEM and AFM. The glass beads with the large diameters were also suitable for examination by AFM because of gentle curvature. Pathogenic bacteria Escherichia coll $0157: \mathrm{H} 7$ (herein after $E$. coil $\mathrm{O} 157: \mathrm{H} 7$ ) were used for demonstration of our microfluidic platform. ${ }^{15,16}$

\section{Materials and Methods}

Microfluidic device design and fabrication. The microfluidic device was fabricated using soft lithography and used here consists of replica molding polydimethylsiloxane (PDMS, Dow-Corning, Cortland, NY, USA) layer and glass substrate ${ }^{17}$ Schematic image of the microfluidic device is presented in Figure 1. The dimension of the microfluidic 


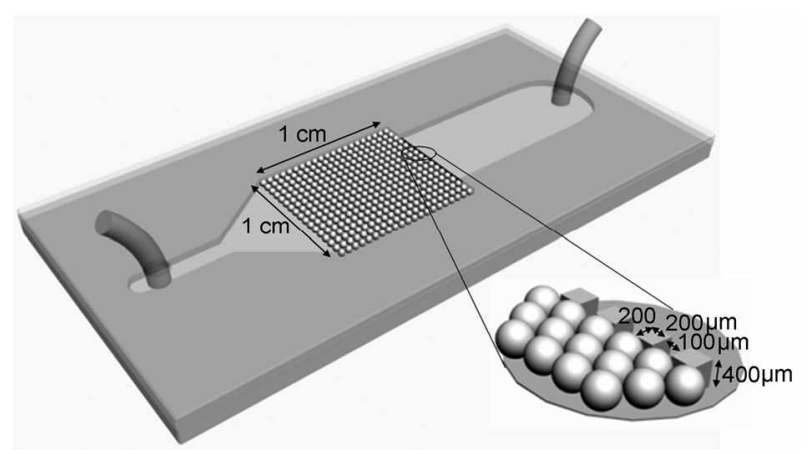

Figure 1. Schematic illustration of the microfluidic device. The microfluidic device constituted of microchannels, microchamber and micropillars. The dimension of the microchamber filled with glass beads was $1 \mathrm{~cm}$ long. $1 \mathrm{~cm}$ wide and $400 / \mathrm{m}$ deep. An array of $200 \mathrm{~mm}$ square micropillars with $100 \mathrm{~mm}$ gap size was situated in the channel to immobilize glass beads. Glass beads in chamber had diameters of about 151 to $212 \mu \mathrm{m}$.

channel was $3 \mathrm{~cm}$ long. $1 \mathrm{~cm}$ wide and $400 \mu \mathrm{m}$ deep and had 1 inlet and 1 outlet, yielding a total volume of approximately $40 \mu \mathrm{L}$. The device consisted of a microchannel and a $1 \mathrm{~cm}$ square microchamber with an array of micropillars, which were developed to immobilize glass beads. It is possible that glass beads immobilize in microchamber, because at the end of microchamber has micropillars which are array of $200 \mu \mathrm{m}$ square micropillars with a $100 \mu \mathrm{m}$ gap size was situated in the middle of the microfluidic channel. The microfluidic device did not need to include weir-structures which were previously used to pack microspheres in other microfluidic devices. ${ }^{18}$ A syringe pump (KD scientific. Holliston, MA. USA) was connected through the tubing for introducing media into channel continuously. Immobilization of glass beads with average diameters in the range of $150-212 \mu \mathrm{m}$ (Sigma Chemical $\mathrm{Co}$.) was initiated by putting glass beads into the channel via the 1 side inlet.

Bacterial strain and growth condition. $E$. coli $\mathrm{O} 157: \mathrm{H} 7$ ATCC 43894 was obtained from the American Type Culture Collection (Manassas, VA, USA). Bacteria was transformed with the gfpmut2 gene encoding plasmid DNA. ${ }^{19}$ The strain was routinely maintained on $\mathrm{LB}$ agar (BD, CA, LSA) supplemented with $100 \mu \mathrm{g} \mathrm{mL} \mathrm{m}^{-1}$ of ampicillin. For biofilm formation, bacteria was grown on M9 minimal medium containing $47.76 \mathrm{mM}$ of $\mathrm{Na}_{2} \mathrm{HPO}_{4}, 22.06 \mathrm{mM}$ of $\mathrm{KH}_{2} \mathrm{PO}_{4}$. $8.56 \mathrm{mM}$ of $\mathrm{NaCl}, 18.7 \mathrm{mM}$ of $\mathrm{XH}_{4} \mathrm{Cl}, 0.4 \%$ of glucose. $0.01 \%$ of thiamine, $2 \mathrm{mM}$ of $\mathrm{MgSO}_{4} \cdot 7 \mathrm{H}_{2} \mathrm{O}$ and $0.1 \mathrm{mM}$ of $\mathrm{CaCl}_{2}$. This preculture was diluted to 100 folds with new $\mathrm{M} 9$ medium and incubated at $220 \mathrm{rpm}$ at $37{ }^{\circ} \mathrm{C}$ to get an optimum culture condition. Microfluidic device was filled with M9 medium prior to inoculation of bacterial cells. When the culture of $E$. coli $0157: \mathrm{H} 7$ reached an $\mathrm{OD}_{600}=$ 0.8-1 (Biowave CO8000. WPA Ltd., Cambridge. England). culture suspension was introduced into microchannel. Flow of fresh M9 media through microfluidic channels was controlled with a syringe pump fitted with specific volume of syringes. Microfluidic device was incubated for $30 \mathrm{~min}$ at room temperature to allow the attachment of the cells with glass beads and microchamber. After attachment periods the fresh media were flown at various flow rates at room temperature.

Staining of biofilm. To visualize the EPS of the biofilm by fluorescence, tetramethylihodamine isothiocyanate (TRTTC)labeled concanavalin A (Sigma Chemical Co), which specifically binds to $d$-( $L$-glucose and $d-(L)$-mannose group on EPS, was used. ${ }^{20} 5 \mu \mathrm{g}$ of TRITC-labeled concanavalin A was resuspended into $1 \mathrm{~mL}$ of phosphate-buffered saline (PBS) and then filtered before use. At first media in channels were washed by PBS with syringe pump and then TRITClabeled concanavalin A was added into channel. After 30 min incubation in the dark at room temperature, remaining staining solution was washed out by flowing PBS. Microscopic observation of stained biofilm was assessed in the microchannel mounted on fluorescent microscope (Axiovert $100 \mathrm{M}$, Carl Zeiss, Jena, Germany). Images were recorded at an excitation wavelength of $488 \mathrm{~nm}$ and emission wavelength of $507 \mathrm{~nm}$ for GFP and at an excitation wavelength of $543 \mathrm{~nm}$ and emission wavelength of $635 \mathrm{~nm}$ for TRITClabeled concanavalin A. Fluorescence intensities were analyzed by Image I program (NIH, LSA).

Scanning electron microscopy (SEM) and Atomic force microscopy. Prior to SEM observations, media and unbounded cells in channels were washed by PBS with the syringe pump. Washed beads were taken out from microchamber and dried for a few hours in air and completely dried by lightly blowing with a pure nitrogen gas. Images were taken by a field-emission SEM instrument (ISM-6700F, JEOL, Tokyo, Japan). Nanoscale topography of biofilms was measured by AFM (Nanofocus Inc., Korea). Sample preparation for the measurement was similar to that used for SEM. ${ }^{21}$ Biofilms were scanned in non-contact mode using a silicon nitride cantilever with resonance frequencies of 204$297 \mathrm{kHz}$ and a spring constant of $50-60 \mathrm{Nm}^{-1}$ in an ambient environment at speed of $1 \mathrm{~Hz}$ with 512 by 512 resolutions.

\section{Results and Discussion}

Biofilm formation in microfluidic device. Figure 2 showed the effect of different flow rates on biofilm formation. Figures 2A-D indicated attached cells marked with GFP and Figures 2E-H indicated biofilms stained with TRITC-labeled concanavalin A. Figure 2I showed the fluorescent intensity of biofilm that was quantified by Image $\mathrm{J}$ and calculated the fold change of basal intensity at $0 \mu \mathrm{L}$ $\mathrm{min}^{-1}$. Fluorescent intensity of attached cells and biofilm at $0.5 \mu \mathrm{L} \mathrm{min}^{-1}$ were $0.9 \pm 0.2$ folds and $1.3 \pm 0.1$, respectively. This result indicated that the growth rate of biofilms formed at 0 and $0.5 \mu \mathrm{L} \mathrm{min}{ }^{-1}$ is similar to each other. The amount of EPS at 5 and $50 \mu \mathrm{L} \mathrm{min}{ }^{-1}$ increased to $4.4 \pm 0.9$ and $2.3 \pm$ 0.2 folds, respectively, than the value of EPS under static condition. The strongest fluorescent intensity was observed at $5 \mu \mathrm{L} \mathrm{min}^{-1}$.

Next, development of biofilm in our microfluidic system was investigated. Figure 3 showed that biofilm in the microchannel increased at 1 day and the maximum amount of biofilm was observed at 5 and 9 days. Both EPS and cell 

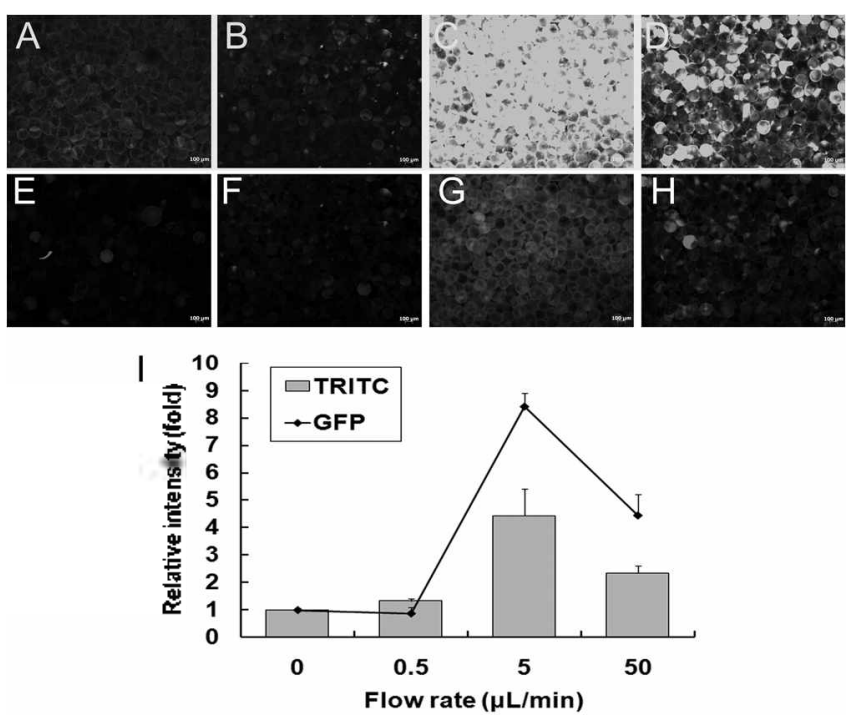

Figure 2. Comparison of biofilm formation at different flow rates; static culture ( $\mathrm{A}$ and $\mathrm{E}$ ). $0.5 \mu \mathrm{L} \mathrm{min}^{-1}$ (B and $\mathrm{F}$ ). $5 \mu \mathrm{L} \mathrm{min}^{-1}$ ( $\mathrm{C}$ and $\mathrm{G}$ ) and $50 \mu \mathrm{L} \mathrm{min}^{-1}(\mathrm{D}$ and $\mathrm{H})$ during 3 days. A-D showed green thuorescence which indicated attached $E$. coli $\mathrm{O} 157: \mathrm{H} 7$ expressing GFP on glass beads. E-H showed red fluoreseence that indicated stained biofilm by TRITC-labeled concanavalin A. (I) Fluorescent intensities quantified with Image J program. Enor bars represent the standard deviations of three independent experiments.

mass which were marked with TRITC and GFP, respectively, steadily increased during the first 5 days. The amounts of EPS at 1,3 , and 5 days increased $10 \pm 0.6,13.9 \pm 0.5$ and $52.9 \pm 3.1$ folds than that obtained at 0 day. Cell mass marked with GFP showed similar trends to EPS. However. after 5 days, both amounts of EPS and cell mass fluctuated possibly due to the deficiency of nutrient and space. It was consistent with the growth curve observed in conventional continuous culture system.22 There was no change in average flow velocities over the fifteen days, indicating that flow was not disturbed by biofilms in the microfluidic channel. Average velocities were estimated from the volume discharged from the device through the outlet every day, suggesting that the device is a suitable for monitoring of

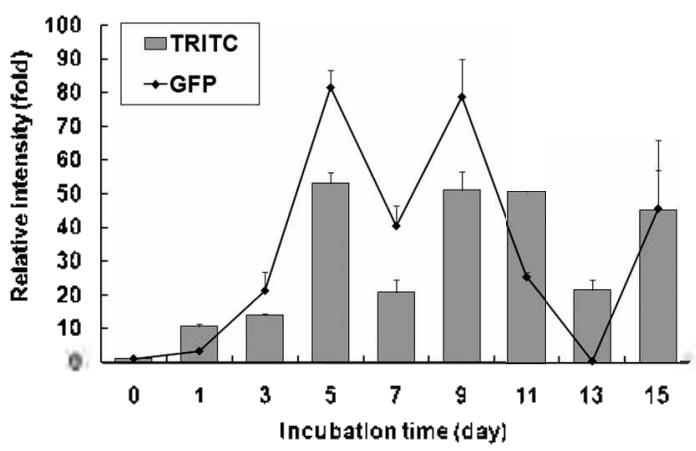

Figure 3. The maturation of biofilm in the microfluidic device. Biofilms were fommed at $5, \mu \mathrm{L} \mathrm{min}^{-1}$ under continuous culture. The GFP indicated attached cells and TRITC showed stained biofilm by TRITC labeled concanavalin A. Fluorescent intensities quantified with Image J program. Error bars represent the standard deviations of three independent experiments.

biofilms over a long-term ( $>2$ weeks).

Morphological study of biofilm using AFM. To demonstrate the effect of fluidic condition on biofilm topography, the differences of biofilm between static and fluidic culture condition were observed by AFM (Fig. 4). AFM has been known to provide sub-micrometer scale spatial resolution of the cellular surface topography as well as nanomechanical characterization of local cellular properties, such as sample elasticity. ${ }^{910}$ Figures $4 \mathrm{~A}-\mathrm{C}$ showed biofilm which grown under stagnant condition and Figures 4D-F were formed biofilm at $5 \mu \mathrm{L} \mathrm{min}^{-1}$ during 5 days. In Figure $4 \mathrm{~A}$, clustering of cells was observed with a few scattered individual cells in static culture condition. However, bacteria covered most surfaces of glass beads in fluidic condition (Fig. 4D). In addition, the surface coverage was measured by applying a threshold so that the biofilms were changed to black and the surrounding channels white using Image J program. The surface coverage of biofilms was the proportional percentage of black to the total area. Figure 4I showed the surface coverage at 0 and $5 \mu \mathrm{L} \mathrm{min}{ }^{-1}$ were $31.6 \pm 8.8$ and $89.4 \pm$ $5.7 \%$, respectively. The surface coverage of cell cluster under static culture was decreased approximately $57 \%$ than
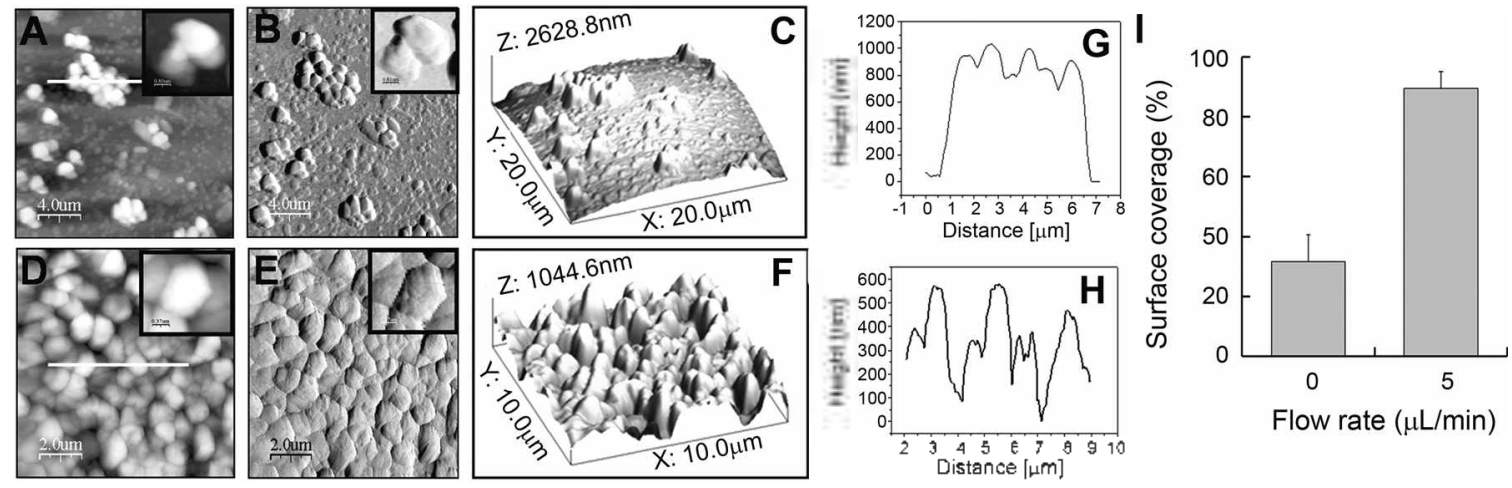

Figure 4. Comparison of biofilms formed under static and fluidic culture conditions after 5 days. (A) AFM topography of biofilm under static culture. Topographic image is $20 \times 20 \mathrm{~m}^{2}$ area. (B) The root mean square (RMS: the standard deviation of $Z$ values) image of Figure 4A. (C) The 3-D topographic image of Figure 4A. (D) Topography of biof $1 \mathrm{~lm}$ formed under flow culture at $5 \mu \mathrm{L} \mathrm{min}^{-1}$. (E) The RMS image of Figure 4D. $(F)$ The 3-D topography of Figure 4D. Inages of small box in Figures A, B, D, and E indicated magnified ones. (G) A topographic line profile obtained from an AFM image of white line in Figure 4A. (H) Line profile obtained from white line in Figure 4D. (I) Quantification of surface coverage done by using Image J program. 
one of cell cluster under fluidic culture at $5 \mu \mathrm{L} \mathrm{min} \mathrm{m}^{-1}$ (Fig. $4 \mathrm{I}$ ). These results suggest that $E$. coli $\mathrm{O} 157: \mathrm{H} 7$ produced more biofilms in fluidic condition than static culture.

In the absence of flow, the cell size width and length were $0.91 \mu \mathrm{m}$ and $1.51 \mu \mathrm{m}$ (Image of small box in Fig. 4B), respectively. However, in case of fluidic condition, cell had $0.72 \mu \mathrm{m}$ of width and $1.1 \mu \mathrm{m}$ of length (Fig. 4E). The average cell size under fluidic condition was slightly smaller than one in static culture. This result seemed to be related with the stress imposed by the flow conditions. ${ }^{5}$ In addition. cells grown under fluidic condition had bumpy surface. This result showed that bacteria produced more EPS under fluidic condition compared to the static condition. The height of biofilms was profiled in Figures $4 \mathrm{G}$ and $\mathrm{H}$. The depth of biofilm in Figure $4 \mathrm{G}$ was almost $1100 \mathrm{~nm}$. In Figure $4 \mathrm{H}$, the two cells of left side was $700 \mathrm{~nm}$ and others between 400 and $500 \mathrm{~nm}$. In addition. Figure $4 \mathrm{H}$ showed bumpy peaks. indicating that the roughness of cell surface was increased by produced EPS.

Morphological study of biofilm in nanoscale using SEM. It is known that the morphology of biofilms is strongly changed between turbulent and laminar flow regimes. ${ }^{23}$ However, there is a limited amount of information about the effect of different laminar flow elements on biofilm morphology. Figure 5 showed biofilm formation under diverse flow rates $\left(0.5-50 \mu \mathrm{L} \mathrm{min}{ }^{-1}\right)$. Interestingly, cell appeared to be stand under 0 and $0.5 \mu \mathrm{L} \mathrm{min} \mathrm{mig}^{-1}$ (Figs. 5A and C). However, biofilms (Figs. 5E and $\mathrm{G}$ ) formed under 5 and $50 \mu \mathrm{L} \mathrm{min}{ }^{-1}$ were thicker and denser than those formed at the lower counterparts (Figs. 5A and C), which is supported by height profile of biofilms (Figs. $4 \mathrm{G}$ and $\mathrm{H}$ ).

In addition, Figures $5 \mathrm{C}$ and $\mathrm{E}$ showed reticular structures on the surface of biofilms formed at 0.5 and $5 \mu \mathrm{L} \mathrm{min} \mathrm{m}^{-1}$ at 3 days. The reticular structures consist of nanopores having diameter ranging from $14 \mathrm{~nm}$ to $100 \mathrm{~mm}$. It is known that biofilm disturbs flow because of high cell densities and EPS. So diffusion is the predominant transport process within biofilm. ${ }^{2+}$ Therefore, nanopores in biofilms may help diffusion of substrate such as nutrient and oxygen for the growth of biofilm embedded bacteria. In static culture condition, nanopores disappeared in biofilms formed at $50 \mu \mathrm{L} \mathrm{min}-1$ (Figs. 5G and H). After 5 days, mature biofilms formed under different flow rates (Figs. 5B, D, F and $\mathrm{H}$ ). In Figures $5 \mathrm{D}$ and $\mathrm{F}$, reticular structures sparsely remained at 0.5 and 5 $\mu \mathrm{L} \mathrm{min}^{-1}$, indicating that pores were clogged gradually as EPS produced. Figure $5 \mathrm{~B}$ showed that biofilms still sparsely presented on the glass beads under stagnant condition. In contrast, biofilms (Fig. $5 \mathrm{H}$ ) formed at $50 \mu \mathrm{L} \mathrm{min} \mathrm{mere}^{-1}$ wer overspread on the entire surface of beads and was denser than that those (Fig. 5G) formed at 3 days. These images
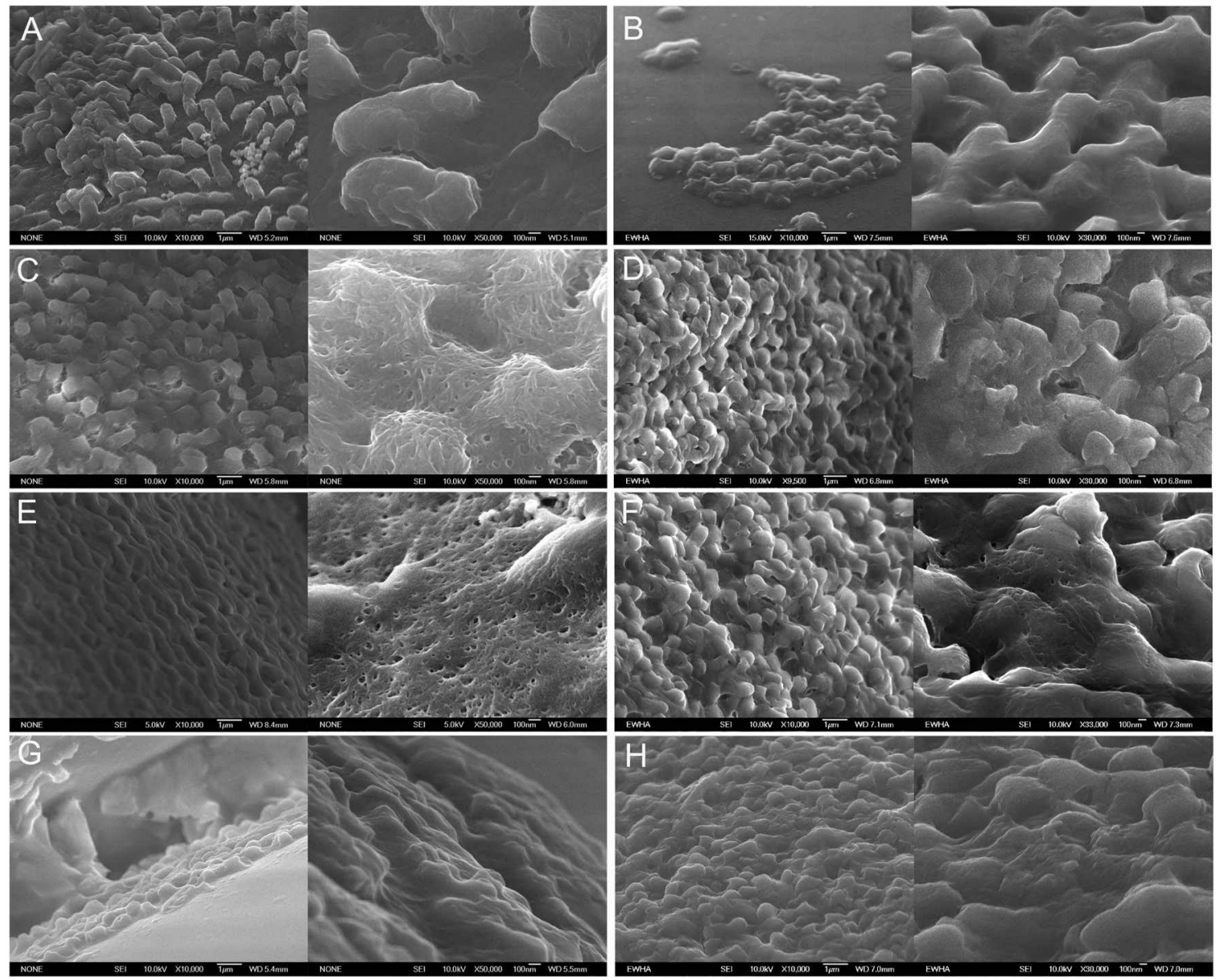

Figure 5. SEM micrographs of biofilms. Biofilms were formed at different flow rate; static condition (A, B), fluidic condition at $0.5 \mu \mathrm{L}$ min ${ }^{-1}$ (C. D). $5 \mu \mathrm{L} \mathrm{min}^{-1}$ (E. F) and $50 \mu \mathrm{L} \mathrm{min}{ }^{-1}$ (G. H) during 3 days (A. C. E. and G) and 5 days (B, D, F, and H). 
(Figs. 5B, D, F, and H) also showed that diminution of pores was caused by increase of shear stress to the biofilm surface according to increase of flow rate, resulting in denser and stronger biofilms. These results suggest that bacteria can respond to flow rates and accordingly change their morphology in biofilm and biofilm structures are thus strongly depending on the hydrodynamic conditions under laminar flow regime.

\section{Conclusion}

Our results showed that bacteria responded to slight change of environmental factors including flow rate and thus altered their architecture of biofilms to adapt their environmental stresses such as flow rate ${ }^{23,25,26}$ The easy observation of biofilms was accomplished by immobilizing glass beads in microchannel. Our microfluidic platform allows the use of a high-resolution with nanoscale and 3D imaging model which offers the potentiality to improve our visualization and understanding of the complex dynamics within biofilms.

Acknowledgments. This work was supported by the Korea Research Foundation Grant funded by the Korean Government (MOEHRD KRF-2005-003-C00150).

\section{References}

1. Costerton, J. W.; Stewart, P. S.; Greenberg, E. P. Science 1999, $284,1318$.

2. O'Toole, G; Kaplan, H. B.; Kolter, R. Anmt. Rev Microbiol. 2000, $54,49$.

3. Potera, C. Science 1999, 283, 1837.

4. Stewart, P. S.; Costerton, J. W. Lancef 2001, 358, 135.

5. Simões, M.; Pereira, M. O.; Sillankorva, S.; Azeredo, J.; Vieira, M. J. Biofoutling 2007, 23, 249.
6. Simões, M.; Pereira, M. O.; Vieira, M. J. Wattr. Sci. Technol. $2003,47,217$.

7. Rani, S. A.; Pitts B.; Stewart, P. S. Antimicrob. Agents Chemother. $2005,49,728$.

8. Kuehn, M.; Hausner, M.; Bungartz, H. J.; Wagner, M.; Wilderer, P. A.; Wuertz, S. Appl. Environ. Microbiol 1998, 64, 4115.

9. Oh, Y. J.; Jo, W.; Yang, Y.; Park, S. Appl. Phis. Lett. 2007, 90 , 143901.

10. Cross, S. E.; Kreth, J.; Zhu, L; Qi, F.; Pelling, A. E.; Shi, W; Gimzewski, J. K. Nanotechnologr 2006, 17, S1.

11. Erlandsen, S. L.; Kristich, C. J.; Dunny, G. M.; Wells, C. L. $J$. Histochem. Crochem. 2004, 52, 1427.

12. Nam, S. W, Van Noort, D.; Yang, S.; Kìn, S. H.; Park, S. Biochip J. $2007,1,111$.

13. Kou, S.; Lee, H. N.; van Noort, D.; Swamy, K. M. K.; Kim, S. H.; Soh, J. H.; Nam, S. W.; Yoon, J.; Park, S. Angent: Chem. Int. Ed. $2008,47,872$

14. Lee, N. Y.; Yang, Y.; Kim Y. S.; Park, S. Bull. Korean Chem. Soc. $2006,27,479$.

15. Buchanan, R. L.; Doyle, M. P. Food Technol 1997, $51,69$.

16. Kim, Y. H.; Lee, Y.; Kim, S.; Yeom, J.; Yeom, S.; Kim, B. S.; Oh, S.; Park, S.; Jeon, C. O.; Park, W. Proteonics 2006, 6,6181.

17. Park, S.; Wolanin, P. M.; Yuzbashyan, A. E.; Lin, H.; Danton, N. C.: Stock, J. B.; Silberzan, P.; Austin, R. Proc. Natl. Acad. Sci. USA 2003, 100, 13910 .

18. Sato, K.; Tokeshi, M.; Kimura, H.; Kitamori, T. Anol. Chen. 2001, 73, 1213 .

19. Commack, B. P.; Valdivia, R. H.; Falkow, S. Gene 1996, 173, 33.

20. Hassan, A. N.; Frank, J. F. Int. J. Food Microbiol. 2004, 96, 103.

21. Oh, Y. J.; Jo, W.; Yang, Y.; Park, S. Ultramicroscopy 2007, 107, 869 .

22. Stoodley, P; Dodds, I.; De Beer, D.; Scott, H. L.; Boyle, J. D. Biofouling 2005, 2l, 161 .

23. Pereira, M. O.; Kuehn, M.; Wuertz, S.; Neu, T.; Melo, L. F. Biotechnol. Bioeng. 2002, 78, 164 .

24. Stewart, P. S. J. Bacteriol. 2003, $185,1485$.

25. Kuchuna, S. L.; O'Toole, G A. Clm: Opin. Biotechnol. 2000, 1 , 429 .

26. Ahimou, F.; Semmens, M. G; Haugstad, G.; Novak, P. I. Appl. Eniron. Aficrobiol. 2007, 73, 2905 . 